\title{
EL DISCURSO PROFESIONAL EN SERVICIOS SOCIALES COMUNITARIOS: UNA APROXIMACIÓN DESDE LA IDENTIFICACIÓN DE REPERTORIOS INTERPRETATIVOS
}

\author{
Professional discourse at community social services: an approach from the \\ identification of interpretative repertoires
}

JaVier Pacheco-Mangas ${ }^{1}$, María de las Olas Palma-García ${ }^{2}$

\begin{abstract}
Resumen
Introducción: la presente investigación está orientada a ofrecer un análisis donde se establezcan los recursos lingüísticos utilizados por los participantes sobre el contenido y alcance de la prestación básica de información y orientación en los servicios sociales comunitarios, tal como la desarrollan los trabajadores sociales. Material y métodos: siguiendo una metodología cualitativa y la utilización del análisis del discurso en la propuesta de Wetherell y Potter (1996) con el empleo de la herramienta analítica de los repertorios interpretativos, se intentarán resaltar aquellos elementos definitorios, estrategias profesionales, valores, normas, prácticas organizacionales, elementos de la cultura institucional, entre otros, que dan forma a los escenarios donde desarrollan su labor los profesionales y que configuran el sistema de servicios sociales comunitarios. Resultados: las entrevistas realizadas a veinticinco trabajadores sociales de la provincia de Málaga muestran cuatro repertorios interpretativos que reflejan la construcción del sistema de servicios sociales por parte de los profesionales implicados: el olvido de lo comunitario, la eterna indefinición del sistema, el elefante encadenado y la escasez agudiza el ingenio. Discusión: se pone de manifiesto cómo se construye un modelo de intervención distante a lo establecido en las normas y códigos éticos a causa de los comportamientos organizacionales e institucionales, que los profesionales intentan minimizar mediante la puesta en práctica de habilidades personales.
\end{abstract}

Palabras clave: servicios sociales comunitarios, análisis del discurso, intervención social, resiliencia, crisis.

\begin{abstract}
Introduction: The present research is aimed at providing an analysis where the language resources used by the participants are established, on the content and scope of the basic provision of information and guidance in community social services, as carried out by social workers. Material and method: following a qualitative methodology, and the discourse analysis model proposed by Wetherell and Potter (1996) with the use of the analytical tool of interpretative repertoires, the defining elements will be highlighted: professional strategies, values, norms, organizational practices, elements of organizational culture, among others, that shape the scenarios where professionals carry out their work and shape the community social services system. Results: interviews with twenty-five social workers in the province of Málaga, show four interpretative repertoires that reflect the construction of the system of social services by the professionals involved: forgetfulness of community, everlasting uncertainty of the system, the chained elephant, and the necessity of the mother of invention. Discussion: it illustrates an intervention model distant from the established model by the standards and ethical codes due to organizational and institutional behavior, which professionals try to minimize by implementing personal skills.
\end{abstract}

Keywords: social services community, discourse analysis, social intervention, resilience, crisis.

Recibido: 29/11/2013

Aceptado: 24/03/2014

Publicado: 01/12/2014

1. Ayuntamiento de Vélez-Málaga. Servicios Sociales Comunitarios. Cl Pescadería Vieja, s/n. Edificio San Cayetano. E-29700 Vélez-Málaga, Málaga, España. E-Mail: fjavierpacheco@gmail.com Teléfono: (00-34) 657192610 Fax: (00-34) 952544118

2. Universidad de Málaga. Dpto. de Psicología Social, Antropología Social y Trabajo Social y Servicios Sociales, Málaga, España. 


\section{Introducción}

La configuración de los servicios sociales

Los servicios sociales en nuestro país han tenido una puesta en marcha compleja. En cuestión de cuatro décadas, hemos pasado de un modelo basado en la asistencia social, discrecional en las obligaciones del Estado y con un marcado carácter benéfico y de control social, hasta la configuración de un sistema de servicios sociales propiamente dicho, ya en el periodo democrático y tras la Constitución de 1978 (Cerdeira, 1987).

Desde la promulgación de las primeras leyes de servicios sociales de las comunidades autónomas en los años 80 en base a las nuevas competencias constitucionales, hasta el actual Proyecto de Ley de racionalización y sostenibilidad de la Administración Local (proyecto LRSAL 121/000058 de 6 de septiembre de 2013), se han sucedido una serie de cambios normativos y estructurales, entre los que destaca el Plan Concertado de Prestaciones Básicas de Servicios Sociales (en adelante Plan Concertado). Según Gutiérrez Resa (2001) con la materialización del Plan Concertado el Estado «pone de manifiesto la intención de ofrecer a todos los ciudadanos unos servicios sociales de bienestar básicos o comunitarios, públicos y gratuitos» (103). Además este logro sin precedentes de entendimiento y compromiso entre los tres niveles de la Administración en todo el Estado, permitió el establecimiento de un catálogo de prestaciones básicas para todo el territorio nacional que comprendía: información y orientación, apoyo convivencial y ayuda a domicilio, alojamiento alternativo y prevención, inserción y cooperación social.

De estas prestaciones, quizá la que más desarrollo ha experimentado y se ha consolidado como el recurso de acceso al resto de prestaciones de los servicios sociales comunitarios y del sistema en general, es la prestación de información y orientación, prestada a través de profesionales del trabajo social. Existen diferentes nomenclaturas para referirnos a esta prestación, aunque la más extendida es la de servicio de información, valoración y orientación [SIVO]. Este mayor desarrollo dentro de los servicios sociales puede deberse al propio objetivo de la prestación, que actúa como facilitador en la difusión de la actividad del sistema de servicios sociales, donde la información tiene un papel fundamental pues «no se puede usar un servicio que no se conoce» (Charroalde, 1996: 41).

\section{La información y orientación en los servicios sociales comunitarios}

Definir el concepto de prestación en el ámbito de los servicios sociales, puede suponer una mayor dificultad respecto a otros sistemas de protección social, 
pues la literatura científica no ha profundizado demasiado en este aspecto. Algunos planteamientos nos muestran como la prestación en este sistema se configura como una estrategia coadyuvante en el proceso de intervención planteado con la persona, familia o grupo y se trata de un proceso de coproducción, ya que para su producción efectiva requiere de la participación de los usuarios, que debe plasmarse en un contrato terapéutico o compromiso de colaboración (Austin, 2002). Adicionalmente, la realidad impone que el trabajo de los servicios sociales esté orientado hacia una población en situación de riesgo, luego, la intervención social que planteemos, deberá ir encaminada a incidir sobre aquellos factores que influyen de manera directa en los problemas o necesidades sociales (Casas, 2012).

En cuanto al contenido de la propia prestación en el caso de la Comunidad Autónoma de Andalucía, nos viene definido por elementos normativos como (a) respuesta a la necesidad de acceso a los recursos sociales y al ejercicio igualitario de los derechos a individuos, grupos e instituciones (Plan Concertado, s.f.), (b) información a los ciudadanos sobre sus derechos y los recursos sociales existentes en el ámbito de los servicios sociales (art. 10.1 LSSA 2/1988, de 4 de abril), y (c) primer nivel de atención de los servicios sociales comunitarios, que se concreta en tareas de información y asesoramiento, valoración y dictamen sobre las demandas planteadas, la derivación hacia el servicio especializado adecuado y la identificación de necesidades para una mejor programación de los servicios sociales (art. 3.3. D 11/1992, de 28 de enero).

\section{El abordaje profesional: enfoques teóricos y factores psicosociales}

El abordaje profesional de la demanda debe llevarse a cabo siguiendo un modelo teórico de referencia. La propia configuración actual del sistema de servicios sociales, con un enfoque eminentemente individual-familiar, predispone a que el modelo diagnóstico o psicosocial sea la metodología de referencia, caracterizándose principalmente por tener en cuenta a la persona en su contexto y plantear un diagnóstico centrado en las fortalezas y las debilidades de la persona para la solución de sus problemas (Du Ranquet, 1993). Se trata de un modelo que pierde eficacia en intervenciones donde el contexto (social, cultural, económico o institucional) tiene un peso importante en la generación de problemas sociales, al primar aspectos propios del individuo sobre los institucionales y sociales.

Otros autores plantean el uso de un enfoque sistémico, que permitan comprender a los profesionales las dinámicas internas y de relación de quienes solicitan su ayuda, identificando de forma clara la red de problemas en la que 
se encuentra inserta la familia (Palomar y Suarez, 1993). El uso de enfoques metodológicos que tienen como base la teoría de sistemas en el ámbito de la intervención psicosocial, ha sido puesto de manifiesto por Navarro (2002), que destaca la eficacia del modelo ecológico, por cuanto permite etiquetar y clasificar las necesidades y problemas sociales teniendo en cuenta el papel que cumplen en cada contexto de relación. La teoría ecológica en trabajo social plantea una práctica profesional del trabajo social abierta, alejada de los centros donde se prestan servicios sociales, pues «son numerosas las áreas ambientales de intervención [...]» (Segado, 2011: 68), que quizá puede suponer un inconveniente en contextos en los que se desarrolle un trabajo social de carácter burocrático, donde el principio de individualización en el abordaje de la demanda se ve dificultado (Guillén, 1993).

El discurso de los profesionales va a estar marcado por la presencia de una serie de construcciones entorno al fenómeno laboral, que también se extiende al resto de ámbitos vitales. Entre estos elementos destacan la resiliencia y el compromiso laboral (engagement) que van a influir en las acciones de los profesionales como factores protectores ante situaciones adversas. Aunque no podemos referirnos a la resiliencia de manera unívoca, algunos autores como Rirkin y Hoopman (1991) la definen como «la capacidad de recuperarse, sobreponerse y adaptarse con éxito frente a las adversidades y desarrollar competencia social, académica y vocacional, pese a estar expuesto a un estrés grave o simplemente a las tensiones inherentes al mundo de hoy» (como se cita en Henderson y Milstein, 2005: 26). En el caso de los profesionales del trabajo social, la promoción de las capacidades resilientes constituye un proceso con el que conviven desde su formación académica facilitando en su periodo de actividad laboral el afrontamiento de acontecimientos adversos y situaciones de crisis (Palma-García y Hombrados-Mendieta, 2013).

El compromiso laboral o engagement se presenta como un constructo desde la psicología definido como un estado motivador positivo y persistente relacionado con el trabajo e integrado por las dimensiones de vigor, dedicación y absorción (Salanova, Schaufeli, Llorens, Peiró, y Grau, 2000; Schaufeli, Salanova, González-Romá, y Bakker, 2002). Aunque existen pocas investigaciones sobre engagement en el colectivo de trabajadores sociales, algunas como la realizada por Manteca y Durán (2012) con profesionales de la acción social demuestran un alto nivel de compromiso laboral en este sector.

Además de estos conceptos positivos, el burnout o síndrome de quemarse por el trabajo es un fenómeno que genera gran preocupación en los contextos donde desarrollan su profesión los trabajadores sociales. Definido por Maslach (1982) como respuesta de los empleados a las situaciones de estrés 
crónico, utiliza una perspectiva tridimensional para su explicación que incluye cansancio emocional, despersonalización y tendencia a la evaluación negativa del propio trabajo. El objeto de la presente investigación no es identificar causas ni plantear respuestas ante situaciones de desgaste emocional, aunque si es interesante tener presente estas dimensiones, pues es posible que puedan presentarse a lo largo del relato discursivo y porque son un problema importante que trasciende el ámbito laboral. En el estudio llevado a cabo por Hombrados-Mendieta y Cosano-Rivas (2013) se pone de manifiesto que el burnout influye negativamente en el apoyo social dentro del trabajo y como la disminución de éste, reduce la calidad de vida fuera del ambiente laboral.

\section{Crisis, reformas y futuro de los servicios sociales}

La recesión económica a nivel global, que en España está teniendo una mayor repercusión, unida al establecimiento de estrategias políticas y económicas de corte neoliberal (Navarro, Torres, y Garzón, 2012), ha desencadenado una serie de cambios en las políticas de protección social y en la configuración y contenido del sistema público de servicios sociales. Según Ramírez-Navarro (2013) destaca la paralización de facto del Sistema de Autonomía Personal y Atención a la Dependencia, con el retraso en la incorporación de los dependientes moderados al 2015 y la eliminación del nivel acordado de financiación; así como la importante disminución presupuestaria llevada a cabo por la administración central desde 2011 en su aportación al desarrollo de prestaciones básicas de servicios sociales (Cáritas Española, 2012).

El Programa Nacional de Reformas del Reino de España (PNRRE, 2013) se configura como el marco sustentador de los planteamientos políticos futuros, algunos de los cuales pueden tener un impacto negativo en la definición de los servicios sociales comunitarios. Entre éstas destaca principalmente la modificación del régimen competencial local, mediante el Proyecto de Ley de Racionalización y Sostenibilidad de la Administración Local (proyecto LRSAL 121/000058 de 6 de septiembre de 2013). Éste plantea una delimitación de las obligaciones municipales en materia de servicios sociales, que circunscribe a la «evaluación e información de situaciones de necesidad social y la atención inmediata a personas en situación o riesgo de exclusión social» (art 1.8), dejando el concepto «servicios sociales» para competencias en este ámbito que ejercerán las comunidades autónomas.

En relación a esto, Muñoz Machado (2013) ha planteado sus dudas en cuanto a la constitucionalidad del principio «una administración, una competencia», presente en el discurso entorno al proyecto de ley, pues la Constitución plantea un ejercicio competencial basado en la cooperación entre los 
distintos niveles de la Administración; idea compartida también en algunos aspectos como la imposibilidad de fijar un coste estándar para los servicios municipales, apuntado por el Consejo de Estado (2013); y por los agentes sociales, que añaden además su preocupación por la pérdida de calidad en los servicios de proximidad y en la externalización de los servicios que ahora se prestan a través de empleados públicos (Comisiones Obreras, 2013; Unión General de Trabajadores, 2013), presentando alternativas y exigiendo su retirada (Consejo General del Trabajo Social, 2013).

El objetivo principal de este trabajo es conocer, los aspectos constructivos del discurso empleado por las trabajadoras y trabajadores sociales que desarrollan tareas de información y orientación [SIVO] en los servicios sociales comunitarios. Se pretende ahondar en la concepción simbólica que los participantes tienen sobre la propia prestación, su alcance y las funciones a desarrollar, haciendo énfasis en las estrategias discursivas usadas por los sujetos para representar la realidad. Además la investigación se orientará hacia la reflexión sobre la actual transformación del sistema público de servicios sociales, por lo que los objetivos adicionales serán (a) trazar una comparación con lo planteado en el discurso y los ideales de la profesión fijados en normas que son de aplicación, planteamientos científicos y académicos consensuados y códigos éticos; (b) analizar los cambios que el paradigma neoliberal está produciendo en el sistema y en el comportamiento de los profesionales; y (c) plantear escenarios futuros provocados por los nuevos modelos de financiación y competenciales que se están desarrollando en el ámbito de los servicios sociales comunitarios.

\section{Material y método}

Acercarnos al estudio de los profesionales que desarrollan su labor en el ámbito de los servicios sociales comunitarios puede ser un ejercicio que podemos abordar desde múltiples ópticas y empleando diferentes metodologías. En primer lugar, se ha centrado el foco de la investigación en aquellos profesionales de este nivel asistencial, que entre otras funciones desempeñan tareas de información y orientación; esta elección responde por un lado a la consideración de canalizador de la demanda que tiene esa prestación básica y por otro, porque supone uno de los campos que mayor visibilidad ha dado al colectivo, considerado el hábitat natural del trabajo social en los servicios sociales comunitarios.

En cuanto al planteamiento metodológico, se ha optado por un análisis del discurso siguiendo la propuesta de Wetherell y Potter (1996), planteada por los autores a través de los conceptos de función, variabilidad, construcción y 
la unidad analítica del repertorio interpretativo. La función, define la orientación del lenguaje hacia la acción; el hablar conlleva funciones como justificar, disculpar o explicar, pero a veces producen efectos que van más allá de la mera descripción y no están directamente disponibles para el analista. En cuanto a la variabilidad, los autores hacen referencia a que un acontecimiento o hecho social puede describirse de diversas formas, en la medida en que la función puede variar; es decir, cierto tipo de funciones, nos pueden llevar a determinadas variaciones en el discurso. Por otro lado, la construcción sirve de guía para el análisis, mostrando los recursos lingüísticos disponibles para la conformación del discurso e insistiendo en el carácter performativo del lenguaje por cuanto este tiene consecuencias prácticas. Por último, la unidad analítica del repertorio interpretativo, establece los patrones de regularidad discursiva que se dan entre los hablantes y que «se pueden considerar como los elementos esenciales que los hablantes utilizan para construir versiones de las acciones, los procesos cognitivos y otros fenómenos» (Wetherell y Potter, 1996: 66).

\section{Participantes}

La investigación se ha llevado a cabo en la provincia de Málaga, seleccionando la muestra desde criterios de heterogeneidad discursiva (Íñiguez y Vázquez, 2008) entre los profesionales que trabajan en servicios sociales comunitarios de la provincia. Para ello se realizó un muestreo donde los participantes debían cumplir los siguientes criterios, accediendo a colaborar con la investigación veinticinco profesionales:

1. Trabajadores/as sociales en activo que tenían como cometido profesional (aunque existieran otros), el desarrollo de la prestación de información y orientación en los servicios sociales comunitarios.

2. Que su actividad profesional se llevara a cabo en centros de servicios sociales comunitarios dependientes de las entidades locales (Ayuntamientos y Diputación) de la provincia de Málaga.

3. Que contaran al menos con cinco años de experiencia profesional, en el desarrollo de tareas relacionadas con la información y orientación en servicios sociales.

4. Que se tratara de profesionales considerados por la comunidad como «testigos privilegiados» del objeto de estudio, en atención a su trayectoria profesional y experiencia. Esta información se ha obtenido a partir de cinco informantes clave que no participaron en el estudio, a los que se les explicó el objeto de la investigación, refiriendo cada uno un número variable de sujetos susceptibles de participar en el estudio. 
En la Tabla 1, puede verse un perfil de los informantes clave que colaboraron sugiriendo sujetos susceptibles de participar en el estudio y en la Tabla 2 se describen las principales características de los profesionales que participaron en la investigación. Para preservar el anonimato de los informantes y los entrevistados, los centros de trabajo se presentan de forma agregada y en base a una distribución territorial en zonas de la provincia (Costa-Este, Costa-Oeste y Norte) además de la Diputación Provincial y el Ayuntamiento de Málaga.

Tabla 1. Perfil de los informantes clave que refieren a sujetos participantes

\begin{tabular}{|c|l|c|c|c|c|}
\hline N. & \multicolumn{1}{|c|}{ Centro de trabajo } & Sexo & $\begin{array}{c}\text { Experiencia } \\
(\mathbf{a n ̃ o s})\end{array}$ & Edad & $\begin{array}{c}\text { Sujetos } \\
\text { Referidos }\end{array}$ \\
\hline 1 & Ayuntamiento de Málaga & Hombre & 13 & 38 & 8 \\
\hline 2 & Diputación de Málaga & Hombre & 24 & 51 & 9 \\
\hline 3 & Ayuntamiento Zona Costa-Este & Mujer & 15 & 56 & 8 \\
\hline 4 & Ayuntamiento Zona Costa-Oeste & Hombre & 26 & 53 & 5 \\
\hline 5 & Ayuntamiento Zona Norte & Mujer & 12 & 44 & 4 \\
\hline
\end{tabular}

Fuente: Elaboración propia

Tabla 2. Distribución y perfil de los participantes según la ubicación del centro de trabajo

\begin{tabular}{|l|c|c|c|c|c|c|c|c|c|c|c|c|}
\hline \multirow{2}{*}{ Centro de trabajo } & \multirow{2}{*}{ Entrevistas } & \multicolumn{3}{|c|}{ Sexo } & \multicolumn{3}{|c|}{ Experiencia profesional (años) } & \multicolumn{5}{|c|}{ Edad } \\
\cline { 3 - 14 } & $\mathrm{H}$ & $\mathrm{M}$ & $>5 ;<10$ & $>10 ;<15$ & $>15 ;<20$ & $>20$ & $<35$ & $>35 ;<45$ & $>45 ;<55$ & $>55$ \\
\hline $\begin{array}{l}\text { Ayuntamiento de } \\
\text { Málaga }\end{array}$ & 6 & 3 & 3 & 1 & 2 & 2 & 1 & 0 & 4 & 2 & 0 \\
\hline $\begin{array}{l}\text { Diputación de } \\
\text { Málaga }\end{array}$ & 8 & 2 & 6 & 3 & 4 & 0 & 1 & 1 & 5 & 1 & 1 \\
\hline $\begin{array}{l}\text { Ayuntamientos } \\
\text { Zona Costa-Este }\end{array}$ & 6 & 0 & 6 & 0 & 1 & 1 & 4 & 0 & 1 & 5 & 0 \\
\hline $\begin{array}{l}\text { Ayuntamientos } \\
\text { Zona Costa-Oeste }\end{array}$ & 3 & 1 & 2 & 1 & 1 & 1 & 0 & 1 & 2 & 0 & 0 \\
\hline $\begin{array}{l}\text { Ayuntamientos } \\
\text { Zona Norte }\end{array}$ & 2 & 1 & 1 & 0 & 0 & 0 & 2 & 0 & 0 & 2 & 0 \\
\hline Total & 25 & 7 & 18 & 5 & 8 & 4 & 8 & 2 & 12 & 10 & 1 \\
\hline
\end{tabular}

Fuente: Elaboración propia 


\section{Procedimiento}

La recogida de datos se ha realizado mediante entrevistas individuales en profundidad realizadas a cada participante. Éstas fueron diseñadas para obtener de los participantes secuencias extensas de texto, en relación a unos ejes temáticos, previamente establecidos, sobre los que giraba la entrevista. En este caso, se estableció una guía temática que pretendía explorar distintos campos de la actividad profesional de los participantes como (a) vocación profesional, (b) definiciones e ideas propias sobre el contenido y las funciones a desarrollar en el servicio, (c) formas de abordaje de la demanda, (d) factores que influyen en la calidad asistencial, (e) labor de empoderamiento ciudadano y promoción de derechos y (f) la percepción del futuro.

A todos los participantes que accedieron a colaborar en la investigación, se les explicó el objetivo y el procedimiento a seguir, así como se les garantizó la confidencialidad y el anonimato. Junto a esto, se recabaron datos de contacto para una posterior devolución de la información, garantizando así el acceso sin restricciones a los resultados obtenidos. Con el consentimiento de los participantes, se realizó una grabación sonora de las entrevistas, para facilitar su posterior transcripción y análisis del discurso contenido en las mismas.

El proceso de categorización y obtención de los repertorios interpretativos se realizó con el apoyo del software Atlas.ti (versión 6). En un primer momento, en la fase textual, la herramienta informática ayudó a organizar la transcripción realizada de las entrevistas en archivos de texto. A continuación se identificaron fragmentos significativos de texto (citas) relacionados con los objetivos de la investigación que permitieran un manejo más asequible de los datos transcritos. A cada segmento de texto se le asignó un descriptor, que de forma sintética ayudara a describir las variaciones en el contenido del discurso que permiten comprender su función. En una segunda fase de análisis conceptual, se realizaron repetidas lecturas meticulosas y se relacionaron los diferentes descriptores obtenidos entre sí para facilitar la observación de regularidades discursivas, que parecen representar la realidad de manera similar. Estas representaciones recurrentes empleadas mayoritariamente por todos los individuos son los cuatro repertorios interpretativos obtenidos.

En cuanto a las posibles limitaciones metodológicas de la investigación, es necesario resaltar que la opción por la entrevista en profundidad como técnica de recogida de datos, nos plantea una serie de inconvenientes, en contraposición al uso de técnicas de tipo grupal, entre los que podemos encontrar problemas potenciales de reactividad, fiabilidad, validez y falta de observación directa o participada (Valles, 1999). Además por economía de tiempo, no se ha podido realizar un procedimiento que permitiera la puesta en común 
con otros investigadores de los repertorios interpretativos identificados, para garantizar una mayor fiabilidad y menor sesgo.

\section{Resultados}

Los repertorios interpretativos obtenidos se corresponden con las pautas o patrones de regularidad que los intervinientes en el discurso mantienen a lo largo del mismo, a pesar de la variabilidad de éste. Se quiere por tanto reflejar, como cada uno de los repertorios planteados se pone en práctica, su importancia en el relato discursivo, así como su relación con los planteamientos teóricos de la profesión y con las normas y códigos éticos que rigen la actuación profesional. Después de analizar los discursos recogidos en las entrevistas, se encontraron cuatro repertorios interpretativos.

1. El olvido de lo comunitario.

2. La eterna indefinición del sistema.

3. El elefante encadenado.

4. La escasez agudiza el ingenio.

\section{El olvido de lo comunitario}

En este repertorio los participantes conciben la intervención que se lleva a cabo en los servicios sociales comunitarios desde una perspectiva individual/ familiar, propia del trabajo social de casos. Como se verá más adelante, esto tendrá también repercusión en otras áreas del propio sistema de servicios sociales. Hay una generalidad en el discurso de los profesionales que identifica el objeto principal de su intervención con el usuario individual y/o familia, habiendo desaparecido del discurso las referencias hacia planteamientos metodológicos con dimensión colectiva, o que tengan como base la intervención con grupos o con la comunidad. Sobre este planteamiento, los profesionales hacen patente que el obviar del discurso la perspectiva de la intervención comunitaria, tiene repercusiones directas en la práctica profesional y en lo que el propio sistema ofrece a sus beneficiarios. En los siguientes fragmentos es posible ver una referencia explícita a ese planteamiento y como se identifican factores de tipo organizacional e institucional, como principales causas de estos comportamientos:

Yo creo que se queda en lo individual. Está configurado para lo individual, más que lo comunitario. Se queda más para la atención individual; parece como si lo comunitario, lo hubiéramos sacado fuera (E-6).

Yo cuando empecé se procuraba el trabajo comunitario, ahora no. De todas formas, con la situación que hay, yo soy mucho de ver in situ a la familia, 
de hacer visitas, de hacerle seguimientos, acompañamientos, etc. Lo que pasa es que me lo impiden ahora; las directrices de los que mandan, que quieren que sea una rata de despacho, una dispensadora de recursos. No le dan valor a lo que se hace (E-11).

\section{La eterna indefinición del sistema}

El discurso toma forma en los aspectos que configuran al sistema, tanto en cuanto al contenido y alcance, su dotación como proveedor de recursos y al espacio que ocupa en relación a otros sistemas de protección. En este sentido, las demandas y expectativas que los usuarios dirigen hacia la institución prestadora de servicios sociales (las entidades locales), tienen un papel principal en la construcción de las actuaciones profesionales que se llevan a cabo en él. Estas demandas, en ocasiones poco definidas, proveen de una conciencia de «servir para todo», que puede dificultar el cumplimiento de los objetivos que los servicios sociales comunitarios tienen encomendados:

Bueno yo creo, que tal como está configurando, el SIVO son unos servicios sociales generales, de base, que la gente vienen aquí a preguntar, lo más peregrino, y de eso, se le informa, se le asesora, bien a nosotros mismos o cualquier otro recurso. Es que es cualquier cosa. Por ejemplo esta mañana, ha venido una mujer porque se le han roto las tuberías de su casa (E-2).

Se hace referencia también, a las diferencias que existen entre los recursos con los que se dota la institución, y lo que sería necesario para afrontar la realidad social. Esta disparidad entre lo real y lo ideal, aparece en el discurso, en no pocas ocasiones, relacionada con la actual situación de recesión económica, pero también se plantea como una cuestión que tiene su base en otros aspectos de la propia construcción histórica, política y social del sistema, entre los que se identifican que tienen que ver con el no reconocimiento del trabajo por cuestión de género, en una profesión feminizada:

Hay mucho desfase entre lo que atendemos, lo que debería ser y lo que ofrece la institución, hay un desfase, ahora por la crisis y antes por otras historias, y siempre hay una excusa para no adaptarnos a una realidad que cambia y que tiene unas necesidades (E-14).

A las políticas y los políticos les da igual, sean de la ideología que sean los servicios sociales, no tienen conciencia social, y somos hablando mal y pronto el culo del mundo, cajón de sastre, no nos valoran. Además en una profesión donde hay tantas mujeres, yo creo que nos valoran menos todavía. Tú te vas a otro departamento donde la mayoría son hombres.... «que si las niñas...»; nada más que la forma de dirigirse. Primero porque somos trabajadoras de lo social, y eso como que no tiene tanto prestigio (E-16).

Alternativas. Cuadernos de Trabajo Social, 2014, pp. 9-28 - ISSN 1133-0473

DOI: 10.14198/ALTERN2014.21.01 
No se ve refrendado en el relato planteado por los profesionales, la función de canalizador de la demanda y de acceso al sistema de servicios sociales, que la normativa y la literatura académica atribuyen a la prestación de información y orientación. Hay pocas referencias hacia la incorporación de otros recursos en la intervención que se lleva a cabo, tanto internos (el resto de prestaciones básicas de los servicios sociales comunitarios), como de otros sistemas de protección social (salud, educación, empleo, etc.), a la vez que se denota una falta de trabajo interdisciplinar y no conectado con los recursos de la comunidad en la que el sujeto de la intervención está inserto, tal como se pone de manifiesto en el siguiente extracto:

Hombre yo entiendo que somos, ofrecemos una información, desde nuestros conocimientos técnicos, no sólo de las prestaciones y de los servicios, que hay sino también de la política que se está implementando, y en ese sentido se supone que somos los expertos en todos esos temas. Aunque en realidad creo que tenemos una idea general de las cosas, muy particular de las prestaciones que damos nosotros, pero nos falta bastante idea de cómo el sistema va fluyendo. Nos falta visión de conjunto (E-5).

\section{El elefante encadenado}

El aumento de la demanda y la carga asistencial de los profesionales del primer nivel de servicios sociales comunitarios, a consecuencia de la actual situación económica y social, es un hecho que está comenzando a hacerse visible. En este aspecto coinciden todos los y las profesionales, que además justifican a través de su discurso, como ese incremento de trabajo, les impide desarrollar y cumplir los objetivos de la profesión. En este aspecto, la frustración por la escasa dotación de recursos del sistema, así como la inexistencia de una programación adecuada por parte de la organización para abordar la situación, tiene una influencia directa en el planteamiento a la hora de abordar las demandas por parte de los profesionales:

La carga de trabajo nos sobrepasa; no se puede coger y atender, atender, atender y no pararte a decir: ¿qué estoy orientando?, ¿qué estoy despachando? Es que eso, «despachamos» prestaciones, esa es mi sensación. Y la gente que con la crisis se está acercando a los servicios sociales, ¿qué le ayudamos? Le orientamos, le escuchamos, le aliviamos, porque no hay para más (E-8).

El agotamiento, el escepticismo y la consideración de ineficacia ante el trabajo llevado a cabo, son dimensiones que predicen el burnout o síndrome de quemarse por el trabajo y que tiene repercusiones directas en el cumplimiento de los objetivos, tanto de la profesión, como los que el sistema de servicios sociales tiene encomendados. Los siguientes extractos ilustran la aparición de estos factores y las implicaciones profesionales que conlleva, a la vez que 
muestra un entorno de trabajo (el sistema de servicios sociales comunitarios), fuertemente instrumentalizado y condicionado por factores de tipo organizacional, institucional y político:

Yo creo que estamos en una actitud de acoplarnos a lo que nos caiga, no realmente de ponernos como tenemos que ponernos. Pero creo que no lo hacemos conscientemente, ientiendes? Es que es tanta la demanda que tenemos y el desborde, que no somos conscientes de que no estamos haciendo realmente lo que tenemos que hacer (E-13).

Yo creo que no nos gusta revolver las tripas, y hay que revolver las tripas. Mira la dependencia, la ayuda a domicilio, la falta de recursos, pero proteste usted, proteste. No somos un revulsivo, y se tendría que ser un revulsivo y hacer ver a las gentes que tiene unos derechos y unas obligaciones y que las instituciones también tienen unas obligaciones que tienen que cumplir, y que deben dar respuesta. Y nos acostumbramos a ser una barrera de contención (E-14).

\section{La escasez agudiza el ingenio}

La falta de recursos para el desempeño de la actividad profesional hace que los profesionales tiendan a sobreponerse a estas carencias mediante la puesta en práctica de las destrezas y herramientas propias de la profesión. Además, la puesta en valor del aspecto humano, a través de habilidades como la escucha activa, la empatía y el compromiso profesional permiten salvar las dificultades que se encuentran en la organización. Estas respuestas resilientes y de compromiso laboral o engagement, permite además que la individualización de la intervención social se configure como un factor clave en la consecución de objetivos de promoción y cambio social, que ponen freno, tanto a la carencia de recursos técnicos y materiales, como a las estrategias de estandarización y de socialización en valores que se consideran ideales:

A veces no se le puede solucionar la problemática, incluso a veces no es posible gestionarle nada, pero la escucha activa es fundamental, el hecho de que se sientan escuchados es el punto más positivo de nuestra profesión. Establecer una relación de ayuda buena, es también muy importante (E-16).

Para mí lo que debería de ser es fomentar la autonomía, promocionar a las personas para que ellas puedas desenvolverse de manera más autónoma y resolver sus problemas por medios propios, porque no va a existir nunca un recurso para cada una de las demandas, y ahora lo estamos viendo (E-12).

El discurso nos muestra también, como los recursos con los que los profesionales se dotan para su trabajo diario, no se limita únicamente a aquellos que pertenecen a la esfera de la institución en la que prestan sus servicios o que están disponibles en otros sistemas de protección social. Los recursos generados en el marco de los nuevos movimientos sociales y por la propia comunidad 
(bancos solidarios de alimentos, redes de consumidores, plataforma de afectados por la hipoteca, etc.), son tenidos en cuenta en el proceso de actuación profesional, como vemos en el siguiente relato:

Y luego lo alternativo también. Hay un montón de historias de redes de autoayuda, de apoyo mutuo, redes de autoconsumo, un montón de redes que se han formado a raíz del 15-M, que a mí me parece muy interesante conocerla para nuestro trabajo con la integración social de la gente. Que la gente se integre, si tú quieres que la gente se integre, tienes que participar en alguna historia de estas, para conocer cómo funciona y como lo aplicas a tu trabajo (E-4).

El autoconcepto de los profesionales como referentes en los procesos de cambio social integral de la persona, familia o grupo, hace que las actuaciones profesionales no se limiten a las estrictamente marcadas por la administración en la que trabajan, para lo que se ponen en prácticas habilidades y destrezas adquiridas fuera de la organización:

Hombre yo creo que es complicado ahora mismo, es un momento muy difícil y hacemos un poco de todo. Aquí hacemos de psicólogo, de trabajador social, de administrativo, de gestor y de banquero, porque hasta los extractos de las hipotecas te los traen para que se los mires. Pero también es complicado dar un servicio de calidad ahora mismo. Porque tenemos muy pocos recursos, en todos los sentidos; personales sobre todo, porque somos muy pocos. (E-23).

\section{Discusión}

Aunque no se puede considerar una afirmación taxativa, el mundo es como lo construimos. A pesar de las diferencias individuales, lo que nos identifica como miembros de un grupo, es perceptible a través del discurso, mediante comportamientos y formas de actuar (pues el lenguaje es una forma de acción), de los que en la mayoría de ocasiones no somos conscientes y además es muy posible que hayan sido fijados por la política y grupos dominantes.

El análisis llevado a cabo permitió obtener cuatro repertorios interpretativos (el olvido de lo comunitario, la eterna indefinición del sistema, el elefante encadenado y la escasez agudiza el ingenio), los cuales han posibilitado la construcción del discurso profesional en torno a la prestación de información y orientación de los servicios sociales comunitarios. Igualmente, ha permitido un acercamiento a la realidad de los profesionales que forman parte del sistema, identificando aspectos que pueden servir de punto de partida para futuras investigaciones para una mejor comprensión del funcionamiento de los servicios sociales comunitarios.

Con los resultados podemos comprobar cómo el trabajo social desarrollado en los servicios sociales comunitarios, ha quedado relegado en el discurso 
a una perspectiva de trabajo individual/familiar, frente al desarrollo de modelos de potenciación comunitaria. De acuerdo con Guillén (1993), esta circunstancia supone el tratamiento de las problemáticas sociales como hechos aislados, que se circunscriben a la esfera del individuo o la familia, dejando de prestar atención al contexto como facilitador en la resolución del problema.

La puesta en práctica de este modelo, puede verse además, como una descarga de responsabilidad por parte de los poderes públicos, que penalizan a los individuos y familias de sus disfunciones sociales que se atienden en el marco de un modelo de atención social residual. Supone además, la vuelta hacia un modelo de asistencia social, que plantea ciertas similitudes con la protección social durante el franquismo y que tal como apunta Cerdeira (1987), se caracterizaba por una protección paliativa a la población, la discrecionalidad en las obligaciones de los poderes públicos y una importante función de control social.

Este comportamiento organizacional e institucional va en contraposición al relanzamiento de la intervención comunitaria en los países de nuestro entorno (Casado y Fantova, 2007), al poner los profesionales de manifiesto una pérdida del interés por el trabajo con la comunidad, y comprobar cómo las directrices políticas y organizacionales se dirigen hacia un modelo de tratamiento individual de las patologías sociales. Esta configuración es una construcción social particular, teniendo en cuenta que la normativa general y específica por la que se rigen los servicios sociales comunitarios, propugna un modelo de desarrollo y competencia social que tiene como base el contexto en el que se desarrolla el individuo.

A pesar de esa concreción normativa, se ponen de manifiesto una serie de factores que afectan a la indefinición del sistema y que distorsionan la actuación profesional y lo que se espera de ellos por parte de la administración en la que prestan sus servicios y por los usuarios potenciales de sus servicios. Surgen elementos que podrían interpretarse como de ambigüedad y conflicto de rol, y que tienen como añadidos la percepción de los participantes de una falta de cultura de planificación y de previsión en la dotación de recursos en las administraciones en las que trabajan.

Los resultados también han mostrado como la cuestión del género en una profesión feminizada como el trabajo social, se configura como elemento a tener en cuenta, por cuanto los profesionales la identifican como un factor que ha podido tener repercusión en el desarrollo y consolidación del propio sistema de servicios sociales, de menor intensidad, en relación a otros sistemas de protección o áreas de la administración pública tradicionalmente ocupadas por hombres. Etzioni (1969 como se cita en Lorente, 2004) expone 
este problema cuando califica a las profesiones feminizadas como «semiprofesiones» lo que provoca un distanciamiento en relación a otras disciplinas con mayor presencia masculina.

A su vez, la prestación de información y orientación que los planteamientos normativos y académicos describen como un elemento canalizador $\mathrm{y}$ «de entrada» al sistema, queda relegada en el relato discursivo a un modelo estandarizado de prescripción de recursos ante una necesidad concreta. Se aprecian pocas referencias al trabajo interdisciplinar, interadministrativo o de colaboración con los recursos de la comunidad, que está en consonancia con los planteamientos encontrados de aplicación de un modelo de intervención eminentemente individual/familiar. En este comportamiento puede incidir la burocracia, en la que el trabajo social «está literalmente [...] inmerso» (Guillén, 1993: 189), y que investigaciones recientes han mostrado como la causante de que los profesionales se vean apartados «del proceso de hacer para conocer (reflexión), priorizando aspectos de inmediatez y de atención (operatividad)» (Ballestero, Viscarret, y Úriz, 2013: 131), donde la interdisciplinariedad, como concepto unido a una intervención holística y de calidad, ya no es necesaria.

No obstante, es necesario destacar cómo a través del discurso se observan estrategias puestas en marcha para minimizar las imperfecciones y carencias a las que se enfrentan los profesionales en su trabajo diario, fruto de la práctica reflexiva como proceso «necesario para generar prestaciones y servicios que puedan responder eficazmente a las necesidades expresadas por los usuarios» (Sicora, 2012: 56). Como resultado, la incorporación de los recursos alternativos y no formales como dotación para su propia intervención, aunque se trata de un comportamiento individual ajeno a las políticas organizacionales e institucionales, supone un comienzo importante para la construcción de modelo de trabajo con perspectiva comunitaria.

Junto a esto, la reforma de competencias de la administración local plantea un nuevo modelo que de llevarse a cabo podría suponer la desaparición del sistema tal y como lo conocemos, tanto en su conceptualización, la responsabilidad pública que sobre éstos se ejerce, la distribución competencial y el riesgo de un proceso de privatización (Uceda et al., 2013). Estamos ante una redefinición competencial que según plantean Miguez, Almeida, y Santiago (2013) «sería probable que hubiese que entender que el ámbito que se identifica como de interés municipal es sólo el de los servicios sociales comunitarios básicos, quedando fuera tanto los servicios sociales comunitarios específicos como los servicios sociales especializados» (9). El desánimo y la falta de credibilidad en el propio trabajo presente en el discurso de 
los profesionales puede hacer muy difícil elaborar una defensa sólida para el mantenimiento del sistema y sus prestaciones.

Por otro lado, el aumento de la demanda y la carga asistencial a la que hacen referencia los profesionales, deben ser factores claves a tener en cuenta en la prevención y detección de enfermedades relacionadas con estresores emocionales e interpersonales que se presentan en el trabajo, como el burnout, aunque debe ser evaluado utilizando la metodología adecuada. Sobre este aspecto Cosano-Rivas, Hombrados-Mendieta y Palma-García (2013) exponen que las investigaciones recientes sobre burnout en el trabajo social en España reflejan un colectivo poco quemado con su trabajo, circunstancia que puede responder al importante componente vocacional de los profesionales que actúa de amortiguador.

El discurso analizado muestra el compromiso laboral y el desarrollo de destrezas y habilidades personales como estrategias de afrontamiento adecuado para minimizar las dificultades que presenta la organización. Entre estas estrategias, la resiliencia en el trabajo social, como proceso dinámico e interactivo que surge de la adversidad, juega un papel fundamental para la generación de buenas prácticas que contribuyan al logro de los objetivos profesionales (Barranco, 2011; Palma-García y Hombrados-Mendieta, 2013)

Por último, a modo de conclusión, los resultados obtenidos han permitido poner de manifiesto cómo se construye un modelo de intervención distante a lo establecido en las normas y códigos éticos a causa de los comportamientos organizacionales e institucionales, que los profesionales intentan minimizar mediante la puesta en práctica de habilidades personales.

\section{Bibliografía}

Austin, D. (2002). Human services management: Organizational leadership in social work practice. Nueva York: Columbia University Press.

BAllestero, A., ViscarRet, J., y Úriz, M. (2013). Funciones profesionales de los trabajadores sociales en España. Cuadernos de Trabajo Social, 26(1), 127-138. doi:10.5209/rev_CUTS.2013.v26.n1.41664

BARRANCO, C. (2011). Buenas prácticas de calidad y trabajo social. Alternativas. Cuadernos de Trabajo Social, 18, 57-74.

Cáritas Española (2012). La reducción del gasto como política: una lectura de las políticas de acción social. Informe sobre los Presupuesto Generales del Estado 2013. 21 pp. Madrid: Equipo de Estudios de Cáritas Española.

CASADO, D., y FANTOVA, F. (2007). Perfeccionamiento de los servicios sociales en España: informe con ocasión de la ley sobre autonomía y dependencia. Madrid: Cáritas Española. 
CASAS, F. (2012). Bienestar social y calidad de vida. Seminario del Máster Universitario en Investigación e Intervención Social y Comunitaria. 7 de diciembre de 2012 (no publicado).

Cerdeira, I. (1987). Los servicios sociales del franquismo a la Constitución. Cuadernos de Trabajo Social, 0, 135-158.

Charroalde, J. (1996). Información y orientación en los Centros de Servicios Sociales. Cuadernos de Trabajo Social, 9, 37-56.

Comisiones Obreras (2013). Informe sobre el Anteproyecto de Ley de racionalización y sostenibilidad de la Administración Local.17 pp. Madrid: Federación de Servicios a la Ciudadanía de Comisiones Obreras. Recuperado el 7 de junio de 2013 de http://www.fsc.ccoo.es/comunes/recursos/99922/1651144-Alegaciones_de_CCOO_al_Anteproyecto_de_Ley_para_la_Racionalizacion_y_ Sostenibilidad_de_la_...pdf

Comunidad Autónoma de Andalucía. Ley 2/1988, de 4 de abril, de Servicios Sociales de Andalucía. Boletín Oficial de la Junta de Andalucía, 12 de abril de 1988, núm. 29. pp. 1323-1328.

Comunidad Autónoma de Andalucía. Decreto 11/1992, de 28 de enero, por el que se establecen la naturaleza y prestaciones de los servicios sociales comunitarios. Boletín Oficial de la Junta de Andalucía, 25 de febrero de 1992, núm. 17. pp. 1018-1020.

Consejo General del Trabajo Social (2013). El Trabajo Social ante la reforma de la administración local. Madrid: Consejo General del Trabajo Social.28 pp. Recuperado de http://cgtrabajosocial.com/files/5244g68hj5s24/el_trabajo_ social_ante_la_reforma_de_la_administracion_local.pdf

Consejo de Estado (2013). Dictamen 567/2013, de 26 de junio, sobre el expediente relativo al anteproyecto de ley de racionalización y sostenibilidad de la Administración Local. Recuperado el 9 de septiembre de 2013 de http://www. boe.es/buscar/doc.php?id=CE-D-2013-567

Cosano-Rivas, F., Hombrados-Mendieta, I., y Palma-García, M. (2013). Situación actual del trabajo social y los trabajadores sociales: burnout y satisfacción laboral. El caso de Málaga. Comunicación presentada en el XII Congreso Estatal del Trabajo social, Marbella (Málaga), 14 al 16 noviembre 2013. Madrid: Consejo General del Trabajo Social.

Du RANQUET, M. (1996). Los modelos en Trabajo Social. Intervención con personas y familias. Madrid: Siglo XXI.

España. Proyecto de Ley 121/000058, de racionalización y sostenibilidad de la Administración Local. Boletín Oficial de las Cortes Generales, 6 de septiembre de 2013, núm. 58-1. pp. 1-32.

Gobierno de España (2013). Programa Nacional de Reformas del Reino de España. Recuperado del 12 de julio de 2013 de http://www.lamoncloa. 
gob.es/NR/rdonlyres/29B5272B-EC30-478C-80F2-B29D675CD4E7/0/ PNREspa\%C3\%Bla2013.pdf

GuilLÉN, E. (1993). La burocratización del trabajo social en intervención social. Cuadernos de trabajo social, 6, 181-193.

GutiÉRREZ RESA, A. (2001). El plan concertado de prestaciones básicas de servicios sociales en España. Revista Española de Investigaciones Sociológicas, 93, 89-130.

Henderson, N., y Milstein, M. (2003). Resiliencia en la escuela (G. Vitale, Trad.). Buenos Aires: Paidós.

Hombrados-Mendieta, I., \& COSANO-Rivas, F (2013). Burnout, workplace support, job satisfaction and life satisfaction among social workers in Spain: A structural equation model. International Social Work, 56(2), 228-246. doi: $10.1177 / 0020872811421620$

ÍNiGUEZ, L., y VÁzQUEZ, F. (2008). Entrevista grupal. Curso de métodos cualitativos de investigación en ciencias sociales. Maestría en Ciencias Sociales. Centro Universitario de Ciencias Sociales y Humanidades. Universidad de Guadalajara. 8-12 de septiembre de 2008. Recuperado el 11 de junio de 2013 de http://www.uv.mx/dgda/files/2013/02/zEntrevista_grupal.pdf

LORENTE, B. (2004). Género, ciencia y trabajo. Las profesiones feminizadas y las prácticas de cuidado y ayuda social. Scripta Ethnologica, 26, 39-53.

MANTECA, A.J., y DURÁN, M.A. (2012). Engagement en profesionales de la acción social. Diferencias entre Administración y Tercer Sector desde la perspectiva del modelo de demandas y recursos laborales. Documentos de Trabajo Social: Revista de Trabajo Social y Acción Social, 51, 45-68.

MASLACH, C. (1982). Burnout: the cost of caring. Nueva York: Prentice Hall Press.

MigueZ, L., Almeida, M., y Santiago, D. (2013). Crisis del estado de bienestar y servicios públicos locales: ¿de los servicios sociales a la asistencia social primaria? Comunicación presentada en el XIX Congreso Italo-Español de Derecho Administrativo, Madrid, 18 al 20 de octubre de 2012. Madrid: Asociación Española de Profesores de Derecho Administrativo.

Ministerio de Sanidad, Servicios Sociales e Igualdad (s.f.). Plan Concertado de Prestaciones Básicas de Servicios Sociales en corporaciones locales 20102011. Madrid. 215 pp.

MUÑOZ MACHADO, S. (4 de julio de 2013). El archivador de las reformas. EL PAIS, Opinión. La cuarta página. Recuperado el 7 de julio de 2013 de http://elpais. com/elpais/2013/06/28/opinion/1372438647_841110.html

NAVARRO, M. I. (2002). La intervención psicosocial con familias multiproblemáticas: la perspectiva ecológica. (Tesis de doctorado). Universidad de Valencia. Recuperado el 11 de junio de 2013 de http://hdl.handle.net/10803/10176 
NAVARro, V., TORRES, J., y GARZÓN, A. (2012). Lo que España necesita: una réplica con propuestas alternativas a la política de recortes del PP (2. ${ }^{\mathrm{a}}$ ed.). Barcelona: Ediciones Deusto.

Palma-García, M., \& Hombrados-Mendieta, I. (2013). The development of resilience in social work students and professionals. Journal of Social Work. Published online 18 March 2013. doi: 10.1177/1468017313478290

PAlOMAR, M., y SuÁrEZ, E. (1993). El modelo sistémico en el trabajo social familiar: consideraciones teóricas y orientaciones prácticas. Alternativas. Cuadernos de Trabajo Social, 2, 169-184.

RAMíREZ-NAVARRO, J. M. (2013). La ley de dependencia en la encrucijada: análisis sincrónico y diacrónico del Sistema de Atención a la Dependencia en España y por comunidades autónomas. (Trabajo de fin de máster). Universidad Internacional de La Rioja. Recuperado el 9 de septiembre de 2013 de http:// reunir.unir.net/handle/123456789/1406

Salanova, M., Schaufeli,W. B., Llorens, S., Peiró, J. M., y Grau, R. (2000): Desde el Burnout al engagement: ¿una nueva perspectiva?, en Revista de Psicología del Trabajo y las Organizaciones, 16(2), 117-134.

Schaufeli, W. B., Salanova, M., González-Romá, V., \& BaKker, A. B. (2002). The measurement of engagement and burnout: A two sample confirmatory factor analytic approach. Journal of Happiness studies, 3(1), 71-92. doi: 10.1023/A:1015630930326

SEGADO, S. (2011). Nuevas tendencias en Trabajo Social con Familias: una propuesta para la práctica desde el empowerment. Madrid: Trotta-UNED.

Sicora, A. (2012). Práctica reflexiva y profesiones de ayuda. Alternativas. Cuadernos de Trabajo Social, 19, 45-58. doi: 10.14198/ALTERN2012.19.03

Uceda, F. X., Martínez, L., Monje, M., Romero, C., Martínez, M., López, A., y GARCíA, M. (2013). Els serveis socials municipals en risc de desaparició: l'avantprojecte de llei de racionalització i sostenibilitat de l'administració local. TS nova: trabajo social y servicios sociales, 7, 73-86.

Unión General de Trabajadores (2013). Valoración y comentarios al anteproyecto de ley para la racionalización y sostenibilidad de la administración local en su versión 18/2/2013. 21 pp. Madrid: Federación de Servicios Públicos de la Unión General de Trabajadores (no publicado).

VAlles, M. S. (1999). Técnicas cualitativas de investigación social. Reflexión metodológica y práctica profesional. Madrid: Editorial Síntesis.

Wetherell, M., y POTTER, J. (1996).El análisis del discurso y la identificación de los repertorios interpretativos. En Gordo, A., y Linaza, J. (comps.), Psicologías, discursos y poder (pp. 63-78). Madrid: Visor. 